the gamma-globulin fraction showed an increase. Regarding the glycoprotein, an increase in the beta-globulin fraction occurred. With regard to the lipoprotein, there was a decrease in the albumin fraction, whereas an increase in the betaglobulin fraction developed.

3. There was a correlationship between the height of the wave from the polarographical filtrate reaction and the alpha-globulin fraction in electrophoreticallyseparated glycoprotein. Changes in the serum protein were discussed on the basis of the above-mentioned findings.

\title{
43. Chemical Research on Neutral Fat and Fatty Acid in Optic Nerves
}

\author{
Jo IMachi, Yoshimasa Isayama, Akio Yamanaka, \\ Takeshi ChIBA AND Keishiro NishizaKI \\ Department of Ophth., Kobe Medical College
}

In order to clarify the dynamic process of chemical changes on demyelination and retrobulbar neuritis, experimental research was made on neutral fat fraction (acetone-soluble fraction), especially on fatty acids analyzed from degenerated dog optic nerves.

Experimental method:-

Degenerated dog optic nerves were examined on the 5 th, 20th, 40th, 60th and 80th day after the exenteration of eyeballs, and as a control, normal optic nerves were also examined. The weight of total neutral fat (acetone-soluble fraction), unsaponifiable substance and fatty acids (i.e. mixed fatty acids) were measured. Mixed fatty acids were analysed by paperchromatography (Bromazon method by Noda), and fatty acids isolated from the tissue of orbit were fractionated and were compared with those of optic nerves. The fatty acids in the optic nerves fractionated by paperchromatography were determined with NodaHirayama method.

The results of experiments were as follows:-

1) The unsaponifiable substances were crystallized as silky lustrous plates; these substances had a melting point of ca $130^{\circ} \mathrm{C}$, and Liebermann-Burchard reactions were positive. Consequently, unsaponifiable substances should be determined as cholesterol.

2) Concentration of total neutral fat increased on the 5th and 40th days. Concentration of cholesterol decreased slightly during the first 20 days and after this increased until 40th day, and then fell rapidly. Concentration of total fatty acids increased steadily throughout the course of degeneration.

3) Composition of fatty acids in normal and degenerated optic nerves was always estimated on a paper chromatogram at definite spots. In the composition 
of fatty acids, stearic, palmitic and myristic acid which belong to saturated acid were detected as spots, oleic and linoleic acid which belong to unsaturated acid were also detected. On the 80th day linolenic acid (unsaturated) was detected. Composition of fatty acids in orbital tissue evidently differed from that of the optic nerves.

4) The results of the quantitative analysis of fatty acids in the optic nerves were as follows:-

a) Concentration of total saturated fatty acid appreciably increased during the first 5 days, and increased gradually until the 60th day, thereafter increased rapidly. Concentration of total unsaturated acids increased at a rate almost similar to the above mentioned saturated until 40th day, but concentration of total unsaturated was lower than that of total saturated. On the contrary, after 60 th day the former was higher than the latter.

b) The rate of increase of total saturated fatty acid was mostly due to palmitic acid and to some extent stearic acid. The rate of increase of total unsaturated was due to mainly oleic acid and to some extent linoleic acid until 60th day, but on 80 th day the chief components of increase were mainly linoleic acid and in some degree linolenic.

\section{A Study of Experimental Head Injury. Its Influence upon EEG of the Limbic System. \\ Torayoshi MukaI, Yukio Ota, Osamu Kusabe AND Yoshiko Ota Department of Neurology, Osaka Municipal University}

An attempt has been made to study the changes in EEG of the limbic system induced by an experimental blow to a skull in the rabbit. The results are as follows:

1) Changes in EEG immediately after the blow: fast activities appear in both leads of neocortex and limbic system (amygdala, hippocampus, piriform cortex, and olfactory bulb). They are most evident in the olfactory bulb.

2) No slow wave is observed until 8-10 minutes after the blow. It was most marked in the neo-cortex.

3) No spike activity appears immediately after the blow. It has no definite correlation with the convulsion induced by the blow.

4) In anesthetized animals barbiturate bursts are observed after the blow.

5) In rabbits killed by EEG blow neocortex disappears earlier than in the limbic system. 